\title{
The potential of asteroseismology for probing the core chemical stratification in white dwarf stars
}

\author{
N. Giammichele ${ }^{1,2}$, S. Charpinet ${ }^{1,2}$, P. Brassard ${ }^{3}$, and G. Fontaine ${ }^{3}$ \\ 1 Université de Toulouse, UPS-OMP, IRAP, 31400 Toulouse, France \\ e-mail: [noemi.giammichele; stephane.charpinet]@irap.omp.eu \\ 2 CNRS, IRAP, 14 avenue Edouard Belin, 31400 Toulouse, France \\ 3 Département de Physique, Université de Montréal, CP 6128, Succursale Centre-Ville, Montréal, QC H3C 3J7, Canada
}

Received 20 October 2016 / Accepted 8 November 2016

\begin{abstract}
Context. The details of the $\mathrm{C} / \mathrm{O}$ core structure in white dwarf stars has mostly remained inaccessible to the technique of asteroseismology, despite several attempts carried out in the past.

Aims. We aim to re-assess the potential of asteroseismology for probing the chemical stratification in white dwarf cores, in light of new highly efficient tools recently developed for that purpose.

Methods. Using the forward modeling approach and a new parameterization for the core chemical stratification in ZZ Ceti stars, we tested several situations typical of the usually limited constraints available, such as small numbers of observed independent modes, to carry out asteroseismology of these stars.

Results. We find that, even with a limited number of modes, the core chemical stratification (in particular, the location of the steep chemical transitions expected in the oxygen profile) can be determined quite precisely due to the significant sensitivity of some confined modes to partial reflexion (trapping) effects. These effects are similar to the well known trapping induced by the shallower chemical transitions at the edge of the core and at the bottom of the H-rich envelope. We also find that success to unravel the core structure depends on the information content of the available seismic data. In some cases, it may not be possible to isolate a unique, well-defined seismic solution and the problem remains degenerate.

Conclusions. Our results establish that constraining the core chemical stratification in white dwarf stars based solely on asteroseismology is possible, an opportunity that we have begun to exploit.
\end{abstract}

Key words. stars: oscillations - stars: interiors - asteroseismology - white dwarfs

\section{Introduction}

For more than two decades, the main outcome of quantitative asteroseismic studies of white dwarf stars has been to derive the fundamental parameters (mass or surface gravity, effective temperature) and the most superficial chemical structure (the H-rich and/or He-rich layer thickness) of these stars (see, e.g., Sect. 8 of Fontaine \& Brassard 2008, and other reviews on the subject from Winget \& Kepler 2008; and Althaus et al. 2010). The deeper structures, in particular the core chemical stratification, have been considered either firstly, as secondary due to the perceived low sensitivity of $g$-modes to these deep regions, or secondly, as features that need to be set a priori, using most notably the results of full evolutionary calculations, in order to perhaps achieve more accurate determinations of the parameters mentionned above (see, e.g., Fontaine \& Brassard 2002; Metcalfe 2005; Castanheira \& Kepler 2008, 2009; Bischoff-Kim \& Metcalfe 2011; Romero et al. 2012; BischoffKim et al. 2014; Giammichele et al. 2016).

However, Córsico et al. (2002) and later on Córsico \& Althaus (2005) showed from detailed studies of mode trapping in two representative white dwarf and pre-white dwarf evolutionary models that some low-order, low-degree $g$-modes can be very sensitive to the detailed structure of the chemical stratification in the white dwarf core. Moreover, recently, Giammichele et al. (2015a, 2016), in a thorough re-analysis of the two pulsating
ZZ Ceti stars and spectroscopic twins GD 165 and Ross 548, quite unexpectedly found that the pulsations detected in GD 165 have only a weak dependence on the bulk composition of the core, while those observed in Ross 548 are quite sensitive to a variation of this quantity. This puzzle found a natural explanation in that all the modes in the derived seismic model for GD 165 have amplitudes and weight functions that do not extend into the deep core, while three of the six modes associated with the periods observed in Ross 548 are partly confined in the core, and therefore bear a strong sensitivity on its composition. These discoveries open up the most interesting possibility that details of the core composition stratification in white dwarf stars could also be probed with asteroseismology using such deeply confined modes. It is with this possibility in mind that we started developing the new numerical tools described at length in Giammichele et al. (2017).

Based on such tools, we assess, in this paper, the potential of asteroseismology for probing quantitatively the core chemical stratification in white dwarf stars, in particular for situations typically encountered in the field when only a relatively small number of modes is available for a seismic analysis. We illustrate in Sect. 2 the typical signature of the core stratification on the oscillations periods and discuss in Sect. 3 a series of tests to evaluate whether this information contained in the period spectrum can be recovered with our approach to white dwarf asteroseismology or not. We conclude in Sect. 4. 

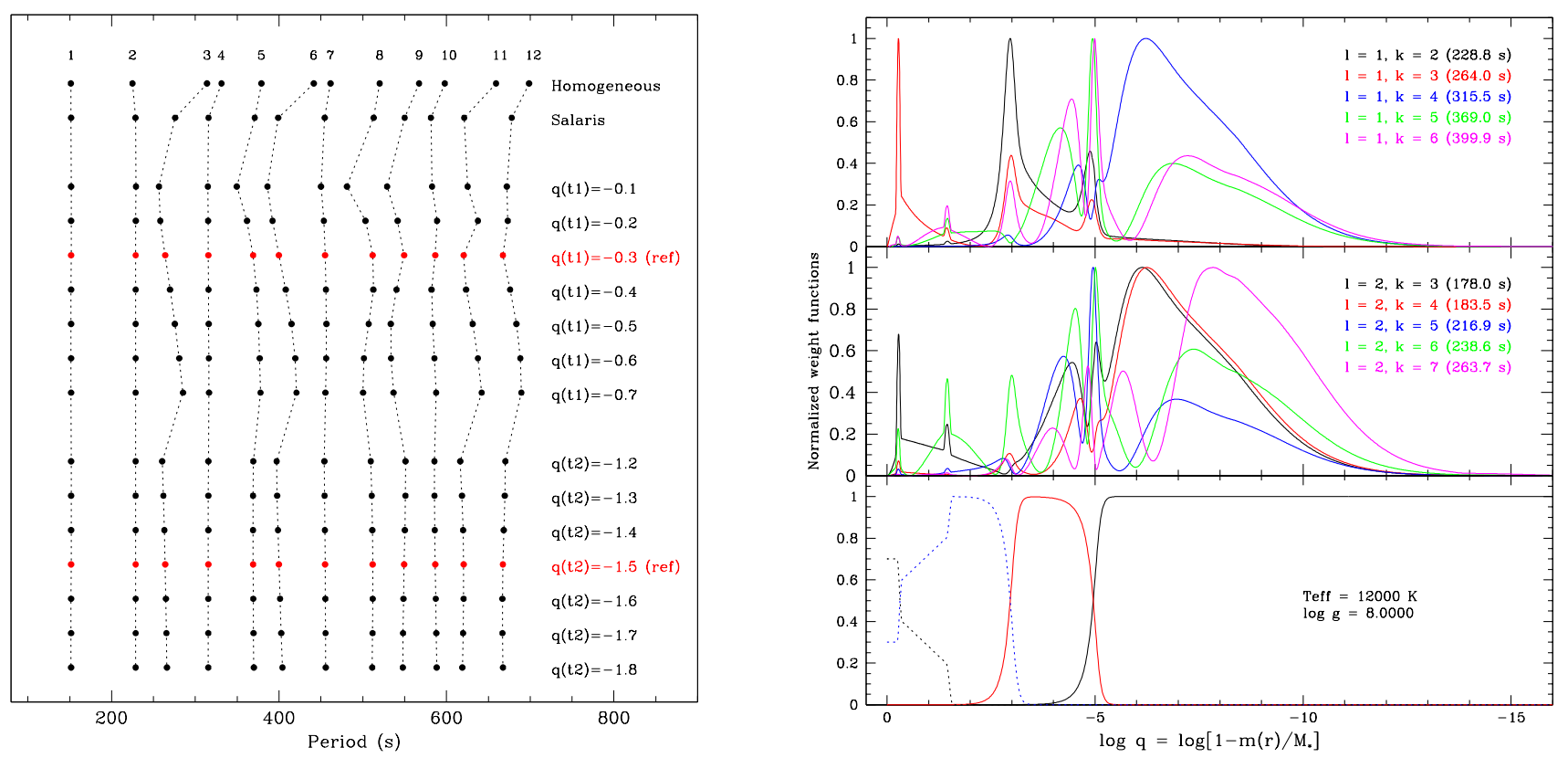

Fig. 1. Left panel: period spectra of the $\ell=1, k=1-12 g$-modes for a series of models with different core configurations (see text for details). Modes of same radial order are connected by a dotted line to ease following period changes. Right panel: oxygen (black dotted line), carbon (blue dotted line), helium (red solid line), and hydrogen (black solid line) stratification in the reference model (bottom panel). The top and middle panels show the weight functions for a subset of dipole $(\ell=1)$ and quadrupole $(\ell=2) g$-modes computed for this reference model.

\section{Seismic signature of the core stratification}

In Giammichele et al. (2017), we introduced a new generation of hydrostatic equilibium white dwarf models designed specifically for in-depth asteroseismic probing of the stellar structure. These new models most notably provide a new parameterization for the $\mathrm{C} / \mathrm{O}$ stratification allowing us to explore a vast range of chemical configurations in the core. In particular, they can closely mimic the double-ramp-like oxygen profiles predicted by standard evolutionary calculations, including cases with a triple transition zone at the core edge (see the details provided in Giammichele et al. 2017). Here, we take advantage of this new tool to characterize the sensitivity of low-degree, low-order $g$ modes (most often observed in white dwarf pulsators) to the core $\mathrm{C} / \mathrm{O}$ stratification. We isolate, in particular, the effects induced by abrupt changes in the core composition.

The left panel of Fig. 1 shows the $g$-mode period spectrum (concentrating on low-order dipole, $\ell=1$, modes) for a series of similar DA white dwarf models, except for their $\mathrm{C} / \mathrm{O}$ core structure. The first two models of the series have, respectively, a homogeneous $\mathrm{C} / \mathrm{O}$ core (75\% oxygen in mass) and a stratified oxygen profile obtained from the evolutionary calculations of Salaris et al. (2010). Important differences in the distribution of the $g$-mode periods between these two models clearly emerge, showing that the impact of the core structure can indeed be significant, an observation in line with the conclusions of Córsico et al. (2002) and Córsico \& Althaus (2005).

With the following models, using the new core parameterization from Giammichele et al. (2017), we identify the main effects perturbing the eigenmode periods. The right panel of Fig. 1 (bottom subpanel) shows the distribution of oxygen (as well as carbon, helium, and hydrogen) in one such models, that we now on refer to as our reference structure. It illustrates the typical double-ramp shape of the oxygen mass fraction characterized by two steep descents. The next seven models represented in the left panel of Fig. 1 reveal how the $g$-mode period spectrum reacts to a change in the position, $q\left(t_{1}\right)$ (also named " $t_{1} \_l q$ " in
Giammichele et al. 2017), of the first drop in the oxygen profile going outward. We find that some modes (e.g., the $k=2$ and $k=4$ modes) remain clearly unaffected, while others (e.g., the $k=3$ mode) experience significant changes of their periods. This is understood by looking at the structure of the modes through, for example, their so-called weight functions (see Brassard et al. 1992a) represented, for our reference model, in the right panel of Fig. 1 (top and middle subpanels). This quantity identifies the regions inside the star that contribute to the period of the mode, thus indicating which part it is sensitive to. In particular, we note that some of the modes have a significant peak arising exactly at the position of the first drop in the oxygen profile (e.g, the $\ell=1$, $k=3$ mode) while others show no amplitude at all there (e.g, the $\ell=1, k=2$ and $k=4$ modes). The latter (or former) are indeed the modes showing no (or a strong) sensitivity to the parameter specifying the position of this transition. We point out that very similarly, though more or less pronounced, peaked structures associated with the chemical transitions between $\mathrm{C} / \mathrm{O}$ and $\mathrm{He}$, and between $\mathrm{He}$ and $\mathrm{H}$, are also seen in these weight functions, thus indicating a specific sensitivity of the modes to the coremantle and mantle-envelope boundaries. This well known sensitivity has indeed already permitted to measure the hydrogen and helium layers thickness in pulsating white dwarfs using asteroseismology. It is due to partial wave reflections at the chemical interfaces causing modes to be either trapped in the upper layers (e.g., in the envelope) or confined below the transitions (e.g., in the core). These trapping and confinement effects, including their impact on the period distribution, have been studied in detail, e.g., by Brassard et al. (1992a). Therefore, we find here that an additional trapping phenomenon is at work due to the deeper chemical gradients expected in the core, which primarily affects modes that are already confined deeper in the star by the other shallower chemical transitions. This trapping effect occurring in white dwarf cores was formerly identified by Córsico et al. (2002) who reported similar structures in the weight functions of some $g$-modes computed from a representative fully evolutionary DA white dwarf model. These authors demonstrated, with 
numerical experiments inspired from Brassard et al. (1992b) and Charpinet et al. (2000), that this effect could have significant impact on the period spectrum. Consequently, there is also a specific signature of the deep core chemical stratification in the period spectrum, which, at least in principle, should be accessible to asteroseismology. This may for instance allow us to place constraints on physical processes such as core overshooting during pre-white dwarf stages, as suggested by Córsico \& Althaus (2005).

The last series of seven models shown in the left panel of Fig. 1 illustrates the impact of changing the position, $q\left(t_{2}\right)$ (also named " $t_{2} \_l q$ " in Giammichele et al. 2017), of the second drop in the oxygen profile. The effect on the period spectrum is obviously less pronounced in this case but a close comparison still reveals some noticeable variations. This lower sensitivity associated to this particular transition is also revealed by the weight functions of the modes (right panel of Fig. 1) that usually only show a small secondary peak in that region. However and most importantly, the variations induced on the $g$-mode frequencies within this series of models can still reach up to $80 \mu \mathrm{Hz}$ (e.g., for the $\ell=1, k=3$ mode whose period varies from $260.5 \mathrm{~s}$ to $266 \mathrm{~s}$ ), which is significantly larger than the precision obtained on measured frequencies, even from relatively short groundbased campaigns. The subtle information relative to this transition is therefore clearly present and, in principle, also accessible to asteroseismic probing.

We end this section by pointing out that because some of the modes, through trapping effects, have a significant sensitivity to the internal structure of the core, models that assume a given stratification, e.g., those obtained from detailed evolutionary calculations, make a rather strong assumption affecting the calculated period spectrum. Even a relatively small shift in the positions of the two oxygen drops would result in period variations that are significant, exceeding largely the precision at which the periods are measured. It can be problematic when such models are used for precise asteroseismic inferences. Unless the real core stratification is very accurately known (though the many uncertainties associated with evolutionary calculations makes this very unlikely), it could potentially bias solutions, or at best limit the accuracy at which the models can match the periods and constrain the other structural parameters of the star.

\section{The potential of asteroseismology}

The specific dependency of the $g$-mode period spectrum to the core chemical stratification discussed above opens up the highly interesting opportunity to constrain the core structure in pulsating white dwarf stars directly from the observed oscillation modes, independently from any evolutionary calculations. To estimate the potential of asteroseismology for that purpose, we propose a series of three tests. We consider our reference model, with parameters and chemical stratification given in the right panel of Fig. 1 (bottom subpanel), as an "artificial star" and compute the pulsation periods associated with this structure. We then apply to each frequency a random perturbation following an unbiased normal distribution with $\sigma=0.1 \mu \mathrm{Hz}$ that mimics typical measurement errors from ground based data. Subsets of the perturbed periods are then selected to form the sets of "observed periods" now considered as if they were typical observations and submitted to our asteroseismic procedure. The latter is described in detail in Giammichele et al. (2016, and reference therein). In a nutshell, it is a double optimization procedure using the forward modeling approach which is solved with an efficient massively parallel multimodal optimization code (named LUCY) based on an hybrid genetic algorithm. This tool allows us to recover efficiently the global solution of the optimization problem, but also any secondary solutions that may occur if the problem turns out to be degenerate. This will be essential as shown below. This code can also deal with high dimension (many parameters) problems, in particular since it does not rely on grid computations (see, e.g., Charpinet et al. 2015), which also is crucial in the present context.

The analyses are conducted using the new core parameterization described and tested in Giammichele et al. (2017). Along with the global parameters $T_{\text {eff }}$ and $\log g$, and the parameters defining the helium and hydrogen distribution, the optimization problem consists of best-matching the set of observed periods with up to 13 parameters to adjust. We stress that most of these parameters are shape parameters allowing to explore with enough flexibility various composition profiles, as explained in Giammichele et al. (2017). The main question arising with this setup is whether the problem is sufficiently constrained to allow for a unique determination of the chemical composition in the core (and elsewhere), or if, on the contrary, the problem remains undetermined with strongly degenerate solutions. The answer certainly depends on the number of observed periods available to constrain the model, as well as the information content of these periods (low-order modes being generally the most significant from that point of view). In the following, we explore three configurations involving, for the first one, a set of ten observed periods, and different sets of five observed periods for the last two experiments. These numbers are not chosen arbitrarily and cover the typical situations that one has to deal with for asteroseismic studies of pulsating DA white dwarfs, since most of these pulsators, especially those close to the blue edge, typically show between five to ten modes only.

The first test is done with ten observed periods, namely the modes $\ell=1, k=2,3,4,5,6$ and $\ell=2, k=3,4,5,6,7$ (all shown in the right panel of Fig. 1). The search for a seismic solution was performed, as usual, on a very wide range of model parameters covering amply all possible pulsating DA white dwarf structures and assuming that only modes of degree $\ell=1$ and 2 are visible. Besides this, no a priori assumption is made on the identification of each mode, which is therefore free during the optimization. The result of the search is summarized in Fig. 2. We find that both the global parameters and the composition stratification of oxygen, carbon, helium, and hydrogen from the original model constituting our artificial star are well recovered at a rather high level of internal precision, according to the probablity distribution functions. We point out here that the derived profiles shown in the top four panels of Fig. 2 are also probability distribution functions of the plotted quantity (normalized to one at maximum) at each $\log q$ position. These are built from the global sampling and evaluation (in terms of best matching the periods) of many different stratifications (virtually several hundred of thousands of models in this specific case) made by the optimization code during the search for the optimal solution. We also find that the periods are well recovered, largely within the errors induced by the random perturbations applied to the original set of frequencies. This first test is therefore extremely encouraging, since a well-defined unique solution is found and recovers correctly the stratification of the star, in particular in the core.

The second test explores the effect of using only five modes for the analysis. We chose the modes $\ell=1, k=2,4,10$ and $\ell=2, k=7,8$ for that purpose. Running the optimization procedure in the exact same manner as before, we find the results summarized in Fig. 3. Again, a unique solution clearly emerges 

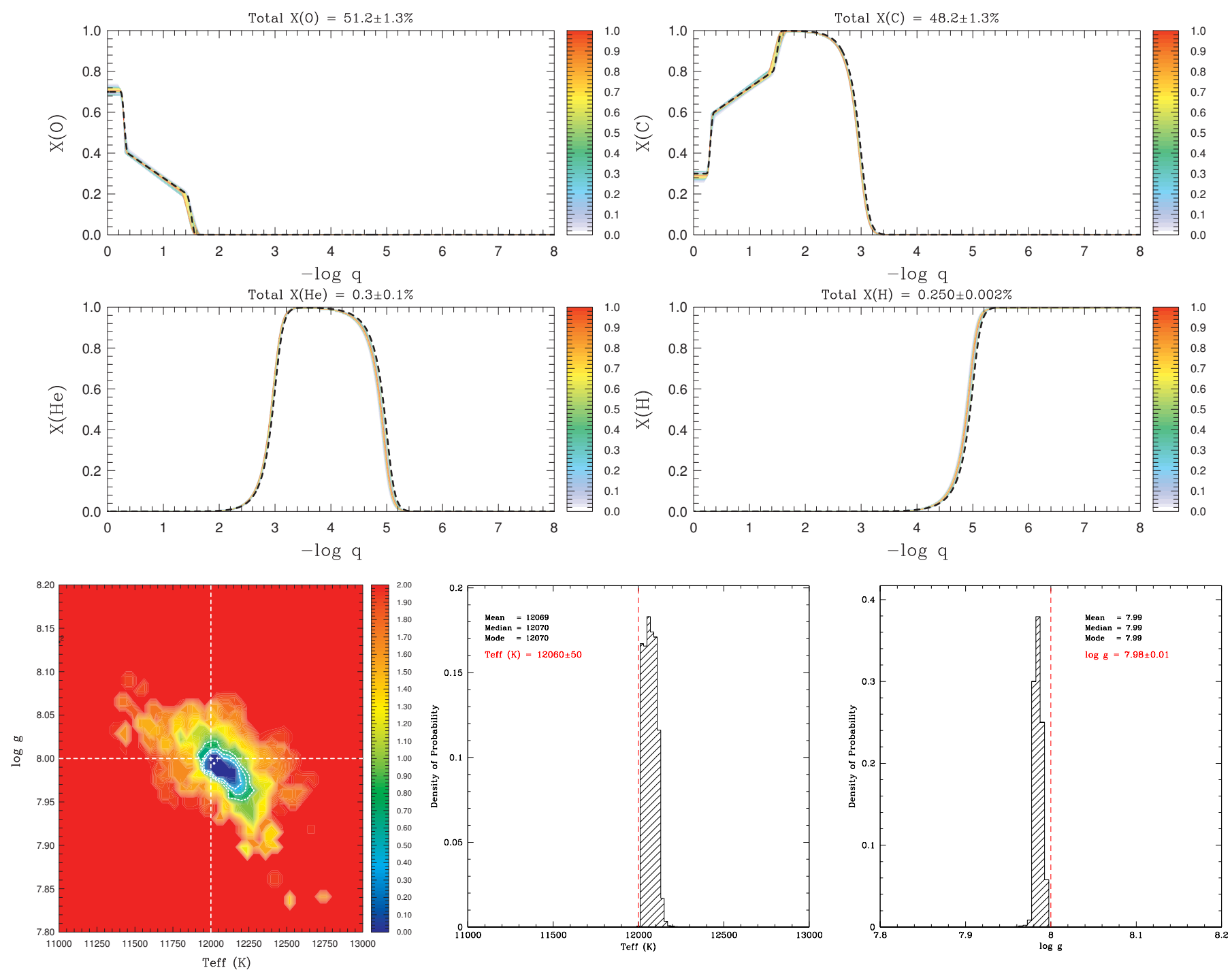

Fig. 2. Seismic solution obtained for our artificial star using ten periods. From top to bottom, left to right, panels show: (1) the derived probability distribution functions normalized for the oxygen, carbon, helium, and hydrogen profiles with, in each case, the true profile from the reference model superimposed as the black dashed curve; (2) the projected $S^{2}$-map (in log scale) for the two global parameters $T_{\text {eff }}$ and $\log g$ with the true values indicated by the white vertical and horizontal dotted lines; and (3) the probability distribution functions derived for these two parameters, with the vertical red dashed line indicating the true value (see text and Giammichele et al. 2016, for further details).

in this analysis, although with an increased spread of the probability distribution functions (larger internal errors). Overall, the original model structure and parameters are very well recovered. We note a small shift particularly in the determination of the position of the second drop in the oxygen profile, which we attribute to a small bias introduced when perturbing the periods randomly. Indeed, even if these perturbations are generated as unbiased normal deviates, we consider here only five periods which represents rather small-number statistics. Moreover, as discussed in the previous section, the sensitivity of the modes to this particular chemical transition is weaker, meaning that a small bias in the period spectrum could more noticeably affect its determination from asteroseismology. The periods are also well matched in that case. This test shows that even with a reduced number of modes, a well-defined seismic solution can be found, allowing us to recover the chemical stratification of the star without ambiguity (but with a lower overall precision). The problem turns out to be still sufficiently constrained in that case.

However, the third and last test of the series underlines that it might not always be the case. This experiment considers another set of five observed periods, namely the modes $\ell=1, k=3,6,8$ and $\ell=2, k=3,6$, which leads to the results illustrated in Fig. 4. The well-defined solution of the former test has now disappeared to leave instead a strongly degenerate set of equivalent solutions, all matching at the highest possible precision the observed periods. This case demonstrates, if need be, that the multimodal optimization code indeed properly finds the multiple solutions when the problem is degenerate. The probability distribution functions are no longer well localized and obviously nothing can be recovered about the original structure of the star. Hence, in this situation the problem is clearly underdetermined, albeit using the same number of modes as the previous test shown in Fig. 3. We point out that some of these degeneracies could probably be lifted, but at the expense of additional constraints or assumptions on the model structure (such as assuming a core oxygen profile, for example).

Finally, we point out that we do not encounter ambiguities in our tests that could be related to an inherent core-envelope symmetry discussed in Montgomery et al. (2003). These authors suggest that a structural degeneracy of the seismic solution may 
N. Giammichele et al.: The potential of asteroseismology for probing the core of white dwarfs
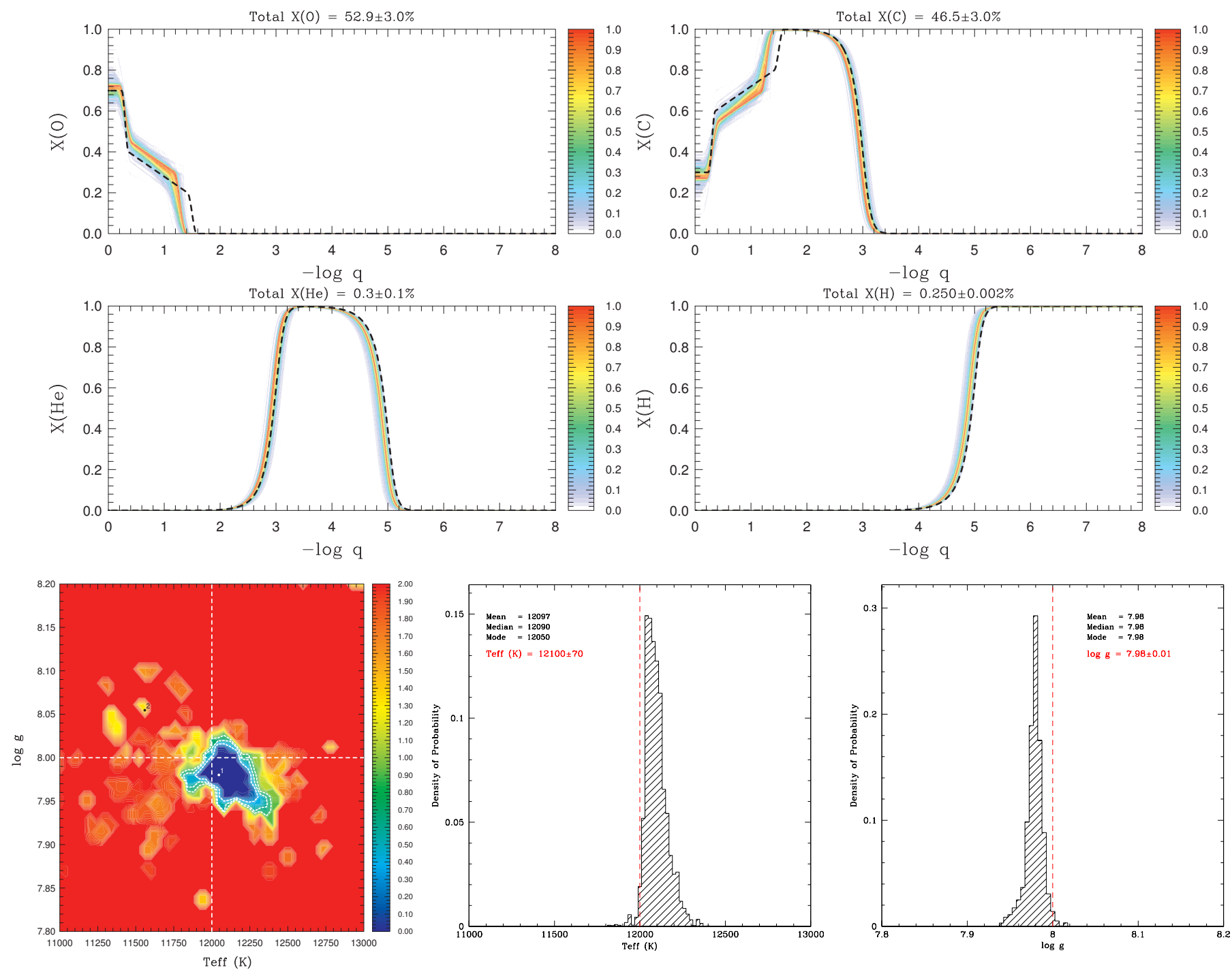

Fig. 3. As Fig. 2, but with a set of five periods that leads to a well-defined solution.

exist due to the way pulsation modes sample the core and the envelope of the star. We clearly do not have this degeneracy in our two well constrained cases where the original model is accurately and unambiguously recovered. We see at least two reasons explaining why this core-envelope symmetry does not manifest itself here. First, the Montgomery et al. (2003) analysis applies to high overtone modes which are in the asymptotic regime, while in our tests, modes of low to mid radial order, typical of ZZ Ceti stars close to the blue edge of the instability strip, are considered. Lower overtone modes are much less affected by this symmetry. Second and more importantly, the core-envelope symmetry is approximate in realistic stellar models, as discussed by the authors themselves. This means that the period distribution in the "symmetrical model" does not exactly match the period distribution of the true stellar structure. In a seismic analysis context, this translates into different values for the merit function quantifying the quality of the fit. Consequently, if a sufficiently accurate period fit can be achieved, the solution corresponding to the true structure would dominate in terms of a significantly better value of the merit function, thus lifting any ambiguity. In the case of the DB pulsator GD358 discussed in Montgomery et al. (2003), the two incompatible best fit solutions proposed by Metcalfe (2003) and Fontaine \& Brassard (2002) have average rms period residuals of $\sigma_{\mathrm{P}} \sim 1 \mathrm{~s}$ which, especially in light of some of the tests carried out in Giammichele et al. (2017), may simply be insufficient to clearly separate the structural signatures of the core and of the envelope. The quality of fit achieved in our present tests and in applications of our approach to real white dwarf pulsators (see, e.g, Giammichele et al. 2015b and forthcoming papers in preparation) is much better and strongly alleviates the risk of such a degeneracy. This underlines the fact that obtaining high fit accuracy, ideally down to the precision of the measured periods, is essential to exploit the potential of probing the core stratification in white dwarf stars from asteroseismology.

\section{Conclusion}

Our examination of the specific sensitivity of low-order, lowdegree $g$-modes to the chemical stratification in the core of white dwarf stars and the series of "Hare \& Hounds" tests allow us to draw two important conclusions about the potential of asteroseismology for deciphering the core properties of these stars. First, we clearly find that the sensitivity of the modes to these deep regions can be exploited to unravel their chemical stratification, even with relatively few modes as often observed for 

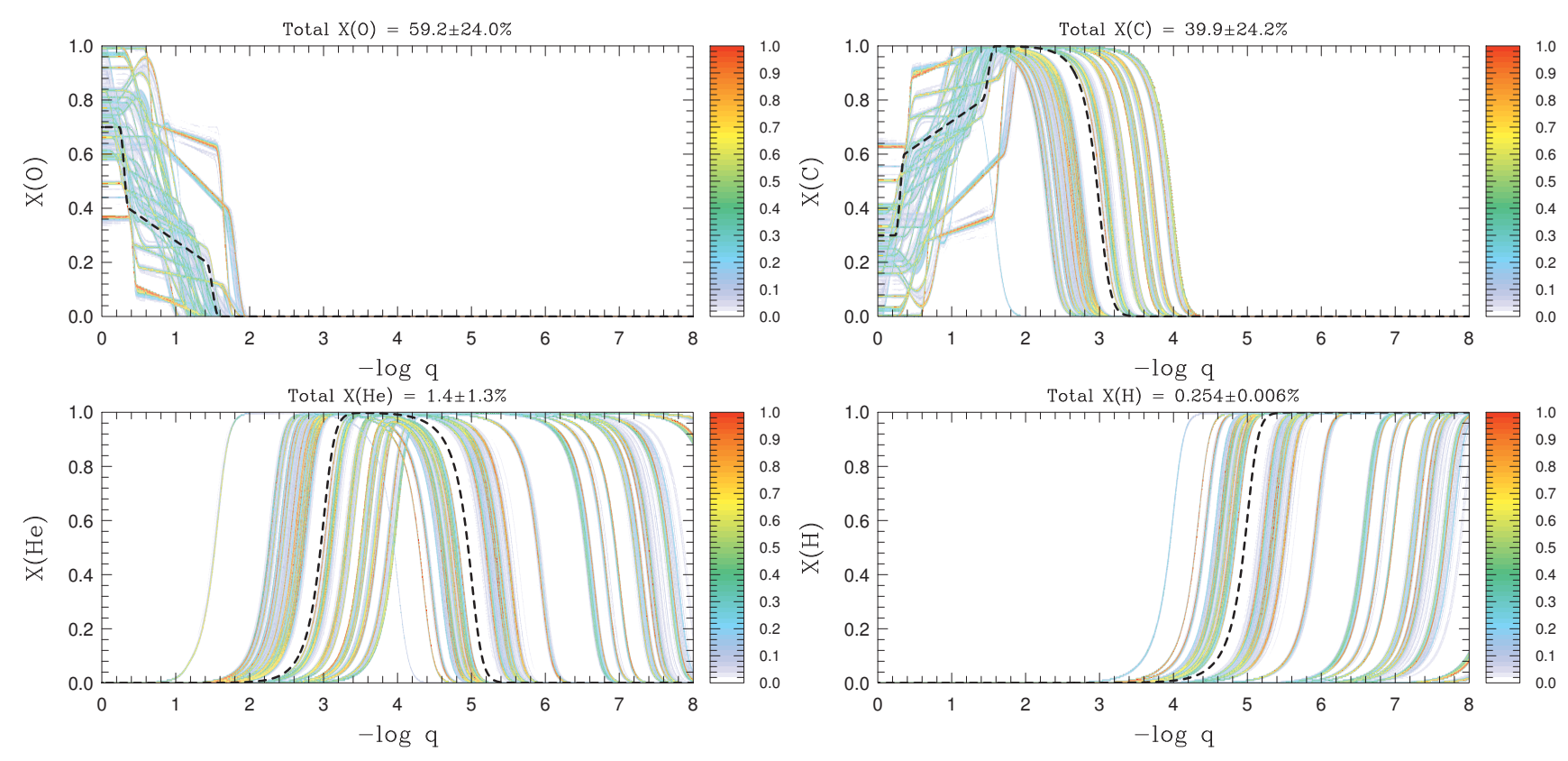
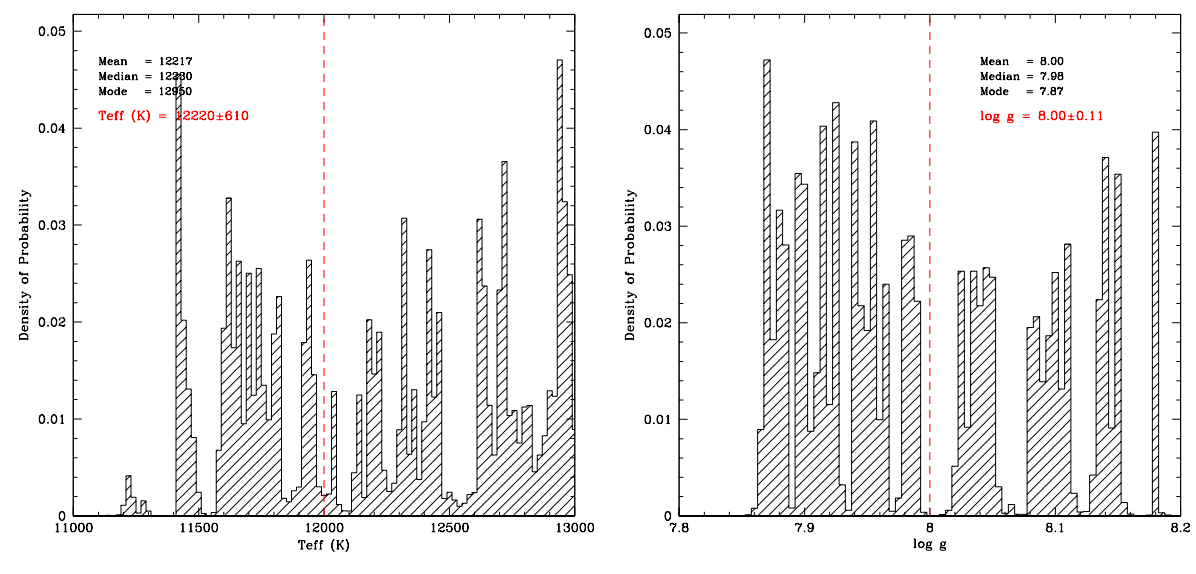

Fig. 4. Same as Fig. 2, but with a set of five periods that leads to degenerate solutions.

pulsating white dwarfs. However, success or failure to do so does not really depend on the actual number of available modes (although having more modes certainly help), but more on which specific modes are observed and used for the seismic analysis. As demonstrated by our tests, the analysis of a white dwarf pulsator showing only 5 modes could as well result in a single welldefined solution unravelling most of its structure or in a useless set of highly degenerate solutions leaving no real clue on the actual structure of the star. This behavior is certainly linked to the individual properties of the modes (illustrated in particular by their weight functions) and the "differential" information contained in the distribution of these modes relative to each other. However, predicting when a set of observed periods leads to strong constraints on the white dwarf core structure remains difficult. Each star should be considered as a specific case and it is only after performing the detailed seismic analysis that it shall be possible to state whether the problem is sufficiently constrained or underdetermined.

We also point out that our tests very clearly refute a statement often heard in the community that models defined with more parameters than the number of observed periods cannot be constrained (i.e., are underdetermined). This widespread misconception of statistics is nicely discussed, e.g., by statistician and Pr. J. Vanderplas in his "Model Complexity Myth" article ${ }^{1}$. We cannot refrain but quote the very first sentences of that article: "An oft-repeated rule of thumb in any sort of statistical model fitting is "you can't fit a model with more parameters than data points." This idea appears to be as widespread as it is incorrect. On the contrary, if you construct your models carefully, you can fit models with more parameters than data points." The faulty assertion in fact holds true only for simple linear systems, such as the well known problem of fitting a set of measurements (of any kind) with polynomials of the type $y(x)$. Note that "linear" here refers to the linearity of model parameters (e.g., the coefficients of a polynomial expression) rather than linearity of the dependence on the data $x$. In a seismic context, the response of the pulsation periods on the variation of one of the defining parameters is, in fact, highly nonlinear and mode-dependent. Hence, the complex optimization problems that we encounter in asteroseismology involving pulsation computations on detailed stellar structures are clearly not linear and do not fit into this class of simple problems.

\footnotetext{
1 https://jakevdp.github.io/blog/2015/07/06/ model-complexity-myth/
} 
Observationally speaking, asteroseismology of pulsating white dwarfs is currently under a revolution with very high quality data delivered from space by the Kepler satellite (original mission) and the still operating Kepler 2 (K2) mission. We also envision that the TESS project will further feed the field with ultra high precision seismic data for many more white dwarfs in a close future. In this context, the potential of asteroseismology for probing the core stratification in white dwarf stars will soon come to life. Detailed analyses of specific objects to achieve this goal have already been briefly exposed, for example, in Giammichele et al. (2015b), and will be fully presented in a series of forthcoming articles.

Acknowledgements. S. Charpinet acknowledges financial support from "Programme National de Physique Stellaire" (PNPS) of CNRS/INSU, France, and from the Centre National d'Études Spatiales (CNES, France). This work was granted access to the HPC resources of CALMIP under allocation number 2016p0205. This work was supported by the FQRNT (Québec) through a postdoctoral fellowship awarded to N. Giammichele. G. Fontaine also acknowledges the contribution of the Canada Research Chair Program.

\section{References}

Althaus, L. G., Córsico, A. H., Isern, J., \& García-Berro, E. 2010, A\&ARv, 18, 471

Bischoff-Kim, A., \& Metcalfe, T. S. 2011, MNRAS, 414, 404
Bischoff-Kim, A., Østensen, R. H., Hermes, J. J., \& Provencal, J. L. 2014, ApJ, 794, 39

Brassard, P., Fontaine, G., Wesemael, F., \& Hansen, C. J. 1992a, ApJS, 80, 369 Brassard, P., Fontaine, G., Wesemael, F., \& Tassoul, M. 1992b, ApJS, 81, 747

Castanheira, B. G., \& Kepler, S. O. 2008, MNRAS, 385, 430

Castanheira, B. G., \& Kepler, S. O. 2009, MNRAS, 396, 1709

Charpinet, S., Fontaine, G., Brassard, P., \& Dorman, B. 2000, ApJS, 131, 223

Charpinet, S., Giammichele, N., Brassard, P., Van Grootel, V., \& Fontaine, G. 2015, in 19th European Workshop on White Dwarfs, eds. P. Dufour, P. Bergeron, \& G. Fontaine, ASP Conf. Ser., 493, 151

Córsico, A. H., \& Althaus, L. G. 2005, A\&A, 439, L31

Córsico, A. H., Althaus, L. G., Benvenuto, O. G., \& Serenelli, A. M. 2002, A\&A, 387,531

Fontaine, G., \& Brassard, P. 2002, ApJ, 581, L33

Fontaine, G., \& Brassard, P. 2008, PASP, 120, 1043

Giammichele, N., Fontaine, G., Bergeron, P., et al. 2015a, ApJ, 815, 56

Giammichele, N., Charpinet, S., Fontaine, G., Brassard, P., \& Zong, W. 2015b, IAU General Assembly, 22, 2256942

Giammichele, N., Fontaine, G., Brassard, P., \& Charpinet, S. 2016, ApJS, 223 10

Giammichele, N., Charpinet, S., Fontaine, G., \& Brassard, P. 2017, ApJ, 834, 136

Metcalfe, T. S. 2003, ApJ, 587, L43

Metcalfe, T. S. 2005, MNRAS, 363, L86

Montgomery, M. H., Metcalfe, T. S., \& Winget, D. E. 2003, MNRAS, 344, 657

Romero, A. D., Córsico, A. H., Althaus, L. G., et al. 2012, MNRAS, 420, 1462

Salaris, M., Cassisi, S., Pietrinferni, A., Kowalski, P. M., \& Isern, J. 2010, ApJ, 716,1241

Winget, D. E., \& Kepler, S. O. 2008, ARA\&A, 46, 157 